\title{
Three-dimensional spiral CT observation of the facet joints of the lower cervical spine and its clinical significance
}

\author{
Qiu An Wang ${ }^{1} \cdot$ Chong Guo ${ }^{1} \cdot$ Ma Ji Sun ${ }^{1} \cdot$ Feng Yuan ${ }^{1}$
}

Received: 20 October 2020 / Revised: 17 December 2020 / Accepted: 17 January 2021 / Published online: 30 January 2021

(c) The Author(s) 2021

\begin{abstract}
Objective By observing the 3D anatomy of normal adult cervical facet joints, using the picture archiving and communication system to measure its $3 \mathrm{D}$ parameters and discussing its clinical significance, the aim of this study was to provide a reliable morphological basis for the design and manufacture of lower cervical facet joint interface distractors.

Methods We selected 200 patients who underwent cervical spine 3D spiral computed tomography (CT) examination in the imaging department of our hospital from September 2019 to May 2020 and whose spiral CT images showed no cervical spinal canal stenosis, cervical disc herniation, obvious bone hyperplasia, or infection. The anterior and posterior diameters of the facet joints on both sides of the cervical spine, the space between the joints, and the left and right diameters were measured on the sagittal, cross-sectional and coronal planes after reconstruction with 3D spiral CT.

Results The anterior and posterior diameters of the facet joints of the cervical spine, the space between the joints, and the left and right diameters all increased from top to bottom along the cervical spine. The 3D parameters of the C2-3 C6-7 segments were significantly different between the male and female groups.

Conclusion The anteroposterior diameter, joint space interval, and left and right diameter of cervical facet joints are different in each segment and between the sexes. The lower cervical facet joint interface fusion device designed according to the measurement results can fully meet the needs of most patients.
\end{abstract}

Keywords Cervical facet joint · 3D spiral CT $\cdot$ Measurement $\cdot$ Interfacial distractor for cervical facet joint

\section{Introduction}

Cervical facet joints (CFJs) are synovial joints formed by the positions of the upper and lower articular processes of adjacent cervical vertebral bodies corresponding to each other $[1,2]$. CFJs maintain the stability of the cervical spine together with the anterior vertebral body and intervertebral discs [3]. The facet joint of the cervical spine forms an angle of approximately $40^{\circ}-45^{\circ}$ with the plane

Feng Yuan

$562622911 @ q q . c o m$

Qiu An Wang

634001275@qq.com

Chong Guo

1535072322@qq.com

Ma Ji Sun

376333460@qq.com

1 Xuzhou Medical University Affiliated Hospital, Xuzhou, China of the anterior vertebral body $2 / 14$. The range of motion of CFJs is relatively large, but CFJs are relatively unstable and vulnerable to injury. These anatomical features of the $\mathrm{CFJ}$ are the anatomical basis for its degenerative changes [4]. In recent years, clinical, imaging, and biomechanical studies have shown that degenerative changes in the facet joints of the cervical spine are one of the important causes of cervical spine instability and nerve root compression symptoms [5-7]. The safest and most effective treatment for the symptoms of nerve root compression caused by degenerative cervical disease is surgery. Although anterior cervical surgery has maintained its undisputed role, the incidence and severity of related complications cannot be underestimated. Surgical wounds and implants may produce large or small postoperative complications, such as the well-known postoperative dysphagia and dysphonia, or clinical manifestations caused by damage to other anatomical structures [8]. Therefore, posterior cervical surgery is a surgical option to ensure effective fusion rate and avoid related complications. ompared with anterior surgery, in the decompression 
process, although muscle dissection is required, posterior approach surgery can still guarantee similar results [9]. With continuous in-depth research on the cervical facet brace, some scholars believe that the cervical facet brace is one of the more ideal ways to treat degenerative cervical spine diseases [10]. Because this method requires the CFJ distractor to be directly placed in the gap of the facet joint during the operation, it is necessary to match the facet joints of each different cervical spine segment, but the anatomy of the facet joints of the cervical spine is complicated. Coupled with the large individual differences, it is often difficult to conduct comprehensive and detailed observations during surgery. Previous research on the facet joints of the cervical spine has mostly been limited to observation on autopsy and of biomechanics [11, 12], and 3D comprehensive descriptions have rarely been reported. In this study, we used advanced 64-row 3D spiral computed tomography (CT) reconstructions of sagittal, cross-sectional, and coronal cervical spine images obtained in the imaging department of our hospital and saved to the picture archiving and communication system (PACS) of our hospital to measure the parameters of the facet joints of the cervical spine in detail, as follows.

\section{Materials and methods}

\section{Clinical data}

From September 2019 to May 2020, 200 patients who underwent a cervical spine 3D CT examination in the spiral CT room of the imaging department of hospital affiliated with Xuzhou Medical University without a history of neck surgery and with no obvious bone hyperplasia, spinal stenosis, intervertebral disc herniation, infection or tumours on the spiral CT images were included. The patients were divided into two groups: the male and female groups, including 100 males and 100 females, aged from 18 to 40 years old (average age: males, 32.08 years old; females, 31.22 years old). The age composition of the male and female groups was the same. For each sample, a total of $103 \mathrm{D}$ parameters of the articular surface of 5 segments from C2-3 to C6-7 were measured. Studies have shown that the facet joints of the cervical spine begin to undergo degenerative changes from the age of 40 , and degenerative changes in the facet joints of the cervical spine are common after the age of 60 [13]. Therefore, this study selected patients under the age of 40 to exclude the influence of degenerative changes caused by age on the measurement of various parameters of facet joints.

\section{Observation and methods of measuring CFJs}

All patients included according to the criteria underwent 3D CT examination of the cervical spine in the imaging

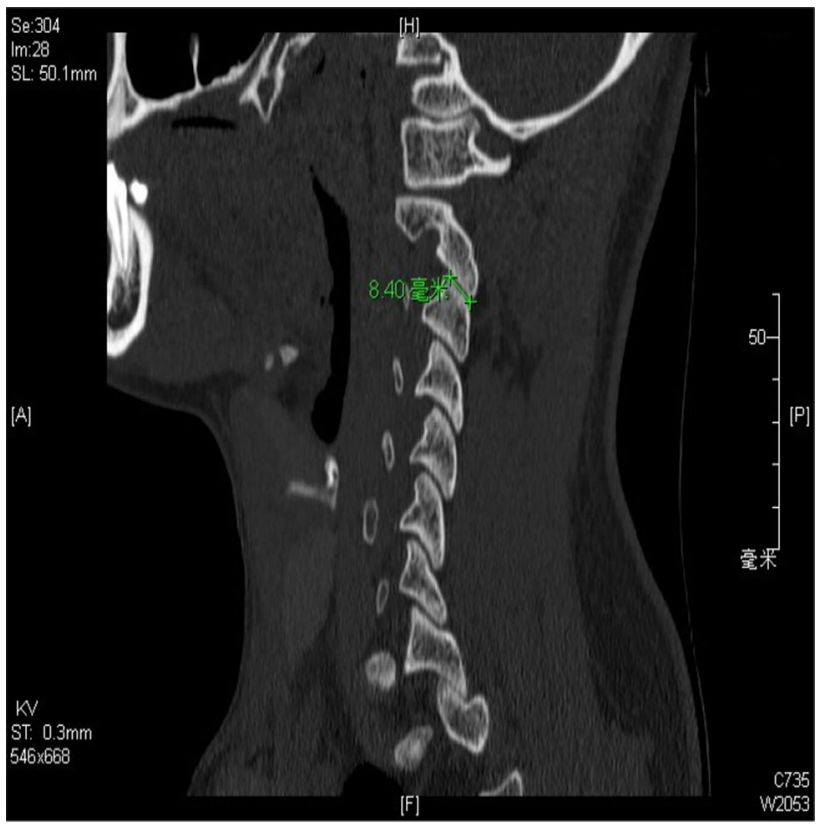

Fig. 1 Measurement of the bilateral anteroposterior diameter of the $\mathrm{CFJ}$ on the sagittal plane

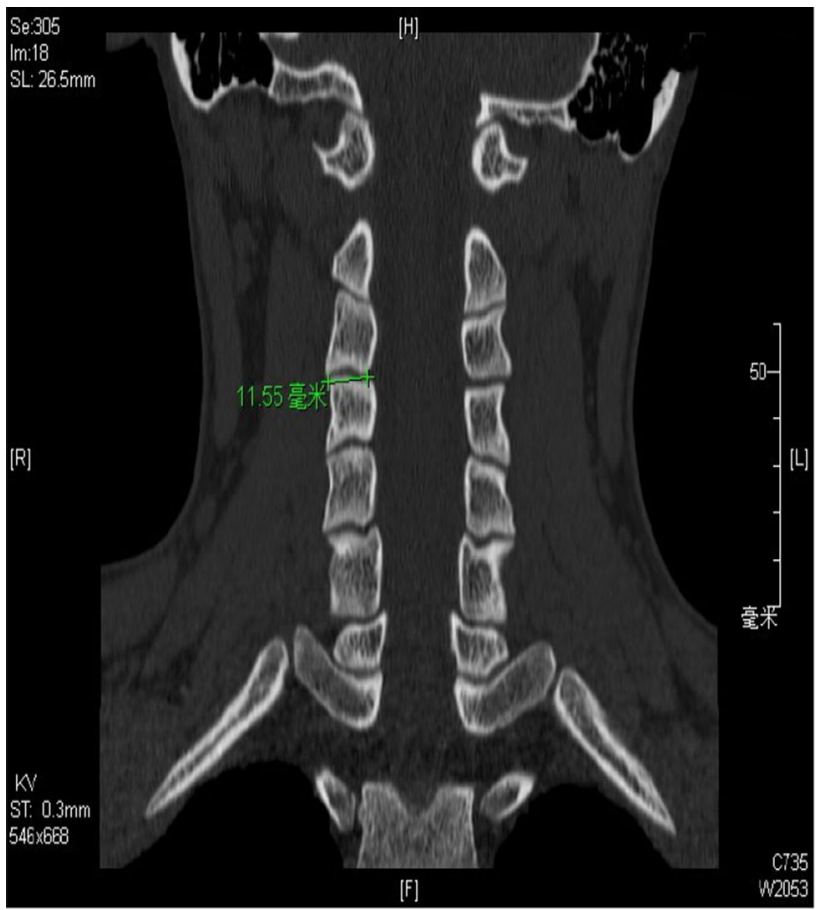

Fig. 2 Measurement of bilateral transverse diameter of CFJ

department of our hospital. The powerful image postprocessing function of the 3D spiral CT system was used to reconstruct the images and transmit the thin-slice data to the PACS of our hospital. All parameters in this study were 


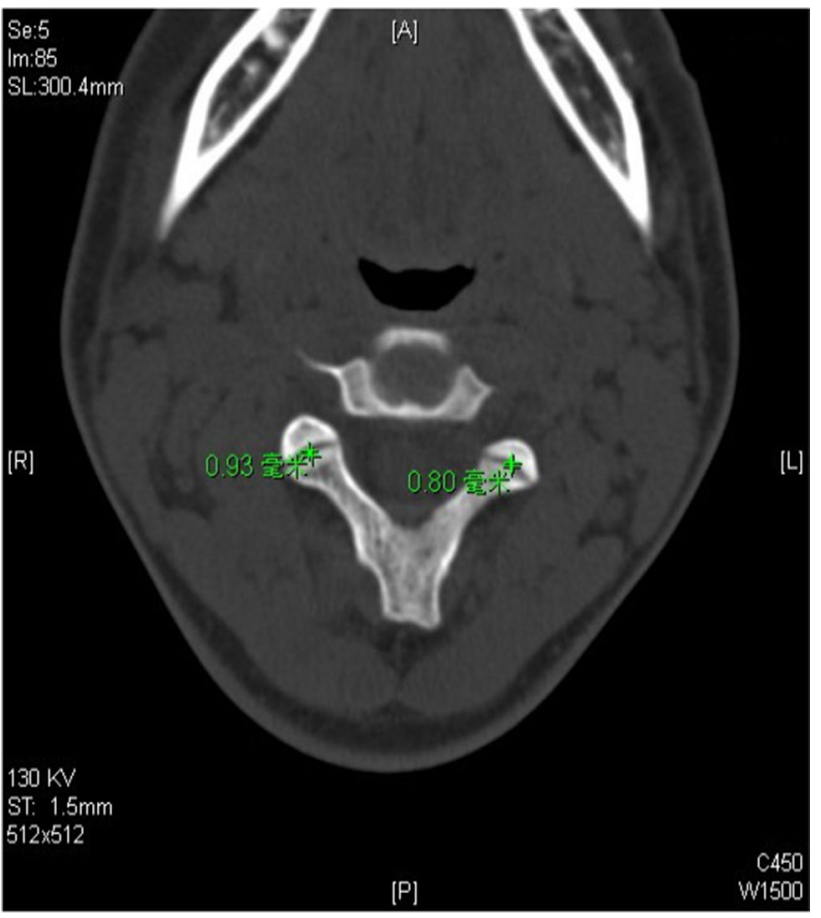

Fig. 3 Bilateral cross-sectional joint gap measurement

manually measured on the window interface of the PACS. The specific measurement method was as follows: after logging in to the PACS, the name or radiation number was entered, and the 3D spiral CT data of the cervical spine were retrieved. The following indicators were measured on the sagittal, cross-sectional and coronal planes of the 3D spiral CT of the cervical spine: (1) The anteroposterior diameter of the CFJs: the largest cross section of the cervical spine on the sagittal plane of the 3D CT image was selected for measurement (Fig. 1); (2) The left and right diameters of the
CFJs: the widest plane of the contact surface of the CFJ on the coronal plane of the 3D CT image was selected for measurement (Fig. 2); (3) The CFJ gap spacing: the cross section of the 3D CT image of the cervical spine was selected for measurement (Fig. 3). Due to the large individual differences in the facet joints and the often unequal widths of the joint gaps, this study selected the widest joint gap for measurement and used the measurement function of the PACS to measure the front and back of the bilateral facet joints C2-3 C6-7 in turn. All bilateral diameter, bilateral joint gap distance and bilateral left and right parameters were recorded after two measurements, and the average value was taken. These values are accurate to $0.01 \mathrm{~mm}$.

\section{Anatomical parameter design of the lower cervical facet joint interface fusion device}

Calculate the mean, standard deviation, minimum and maximum values of measurement indexes such as the bilateral anteroposterior diameter of cervical facet joints, bilateral cross-sectional joint gap, and bilateral transverse diameters of facet joints. The length of the left and right diameter of the cage depends on the measurement of bilateral transverse diameter, the height of the cage depends on the bilateral cross-sectional joint gap measurement, and the front and back length of the cage depends on the measurement of the bilateral anteroposterior diameter of the CFJ on the sagittal plane.

\section{Statistical processing}

The measurement, reading and recording of all parameters were independently completed by the first author on the PACS of our hospital, and the same window width, window level, and magnification were selected during measurement.
Table 1 Comparison of the sagittal plane diameters of the CFJs in Males and Females $(\mathrm{mm}) ;(x \pm s, \mathrm{~mm})$

\begin{tabular}{llllll}
\hline Segments & Males & Females & $F$ & $t$ & $P$ \\
\hline C2-3 & $9.8619 \pm 1.2505$ & $9.10817 \pm 1.1616$ & 0.170 & 4.417 & 0.016 \\
C3-4 & $11.0118 \pm 1.1975$ & $10.3253 \pm 1.1248$ & 0.199 & 4.179 & 0.004 \\
C4-5 & $11.2610 \pm 1.2579$ & $10.3563 \pm 1.0516$ & 1.018 & 5.873 & 0.013 \\
C5-6 & $11.2913 \pm 1.2657$ & $10.3823 \pm 1.0160$ & 7.926 & 5.601 & 0.009 \\
C6-7 & $11.6185 \pm 1.3366$ & $10.9160 \pm 1.3163$ & 0.001 & 3.742 & 0.024 \\
\hline
\end{tabular}

Table 2 Comparison of the CFJ gap in males and females (mm) $(x \pm s, \mathrm{~mm})$

\begin{tabular}{lllrrr}
\hline Segments & Males & Females & $F$ & $t$ & $P$ \\
\hline C2-3 & $0.9543 \pm 0.2688$ & $0.7653 \pm 0.1996$ & 8.766 & 5.644 & 0.003 \\
C3-4 & $1.2122 \pm 0.2778$ & $0.9183 \pm 0.1909$ & 8.385 & 8.937 & 0.010 \\
C4-5 & $1.2603 \pm 0.2584$ & $1.0867 \pm 0.2068$ & 6.650 & 5.245 & 0.002 \\
C5-6 & $1.2734 \pm 0.2807$ & $1.1039 \pm 0.2096$ & 3.877 & 4.838 & 0.034 \\
C6-7 & $1.2878 \pm 0.2737$ & $1.1251 \pm 0.1639$ & 14.599 & 5.100 & 0.016 \\
\hline
\end{tabular}


All measurement data were statistically processed by SPSS 24.0 software, and the data are expressed as $\bar{x} \pm s$. The measurement data were compared using an independent-sample $t$ test, and $P<0.05$ indicated that the difference was statistically significant.

\section{Results}

\section{Bilateral sagittal plane diameter measurements}

The bilateral sagittal plane diameters of the CFJs of C2-3 C6-7 gradually increased from top to bottom. Among them, the C6-7 segment was the largest, at $11.6185 \pm 1.3366 \mathrm{~mm}$ in men and $10.9160 \pm 1.3163 \mathrm{~mm}$ in women. The sagittal plane diameters of $\mathrm{C} 2-3 \sim \mathrm{C} 6-7$ were significantly different between men and women, with both larger in men than women (see Table 1).

\section{Bilateral cross-sectional CFJ gap spacing}

The C2-3 C6-7 CFJ gaps were significantly different between men and women, and the parameters of each segment were greater in men than women. The parameters of each segment of C2-3 C6-7 gradually increased from top to bottom. Segment C6-7 was the widest, at $1.2878 \pm 0.2737 \mathrm{~mm}$ in males and $1.1251 \pm 0.1639 \mathrm{~mm}$ in females (Table 2).

\section{The bilateral transverse diameter of CFJ in coronal on the coronal plane}

The transverse diameter of CFJ in coronal of the articular surface of the cervical spine, from C2-3 C6-7, gradually increased from the top to the bottom of the cervical spine. The difference in the transverse diameter of CFJ in coronal between males and females was statistically significant; the parameters were larger in males than female. Among them, C6-7 had the largest left and right diameters, at $12.3482 \pm 1.0762 \mathrm{~mm}$ in men and $11.5363 \pm 1.1368 \mathrm{~mm}$ in women (Table 3).

\section{Discussion}

In this study, 3D spiral CT in our hospital was used to obtain $3 \mathrm{D}$ reconstructions of the cervical spine $\mathrm{CT}$ of the included patients. After obtaining sagittal, cross-sectional, and coronal images, the parameters were measured through the measurement tools of the PACS at our hospital. Previous observations and measurements of the CFJs have mostly been limited to the study of the anatomy of cadaver specimens, while imaging studies of the CFJs have been rare and have mainly focused on X-ray and conventional CT images [14, 15]. However, on X-ray examination, the images are 2D, and conventional cervical CT examination can only provide cross-sectional images and cannot fully display the shape of the CFJs, as can 3D spiral CT. In recent years, with the widespread use of 3D spiral CT in clinical practice, the ability to collect true volumetric data has been achieved, which not only guarantees the quality of the scanned images but also improves the spatial resolution of and postprocessing functions that can be applied to the scanned images. In 3D spiral CT, the original scanned data can be processed twice to yield more detailed 3D reconstructions. This method can not only display the shape of the skeletal system and its surrounding structures from multiple angles and multiple planes but can also reproduce the volume intuitively in a 3D manner. In terms of anatomical relationships, the measurement function of the PACS can be used to more conveniently perform fine and accurate anatomical measurements of the CFJs on the sagittal, cross-sectional, and coronal planes, overcoming the limitations of X-ray and conventional CT examinations, which can only present CFJs at a single angle, ultimately resulting in observations with improved accuracy [16-18]. In this study, the original 3D spiral CT images of different segments in male and female patients were used for $3 \mathrm{D}$ reconstruction. According to the above methods, the facet joints were comprehensively, carefully, and accurately observed in three dimensions. The 3D parameters of all CFJs were measured with the aim of more comprehensively reflecting the impact of the CFJs on the overall stability and mobility of the cervical spine and facilitating the future production of CFJ interface distractors by providing real and reliable data.
Table 3 Comparison of the transverse diameter of $\mathrm{CFJ}$ in coronal in Males and Females $(\mathrm{mm})(x \pm s, \mathrm{~mm})$

\begin{tabular}{llrlll}
\hline Segments & Males & \multicolumn{1}{l}{ Females } & $F$ & \multicolumn{1}{l}{$t$} & $P$ \\
\hline C2-3 & $10.0073 \pm 1.1675$ & $9.3165 \pm 0.9653$ & 3.969 & 4.560 & 0.019 \\
C3-4 & $10.3782 \pm 1.1246$ & $9.5415 \pm 1.0593$ & 0.175 & 5.416 & 0.006 \\
C4-5 & $10.7201 \pm 1.1304$ & $9.9017 \pm 0.9720$ & 1.933 & 5.489 & 0.021 \\
C5-6 & $11.3952 \pm 1.2677$ & $10.3979 \pm 1.0664$ & 4.210 & 6.020 & 0.001 \\
C6-7 & $12.3482 \pm 1.0762$ & $11.5363 \pm 1.1368$ & 0.002 & 5.186 & 0.004 \\
\hline
\end{tabular}


Since Denis [19] first proposed the "three-column theory" of the spine in 1983, many scholars have confirmed that the facet joint is an important bony structure of the posterior spine $[5,6]$ for maintaining the overall stability of the cervical spine and ability to perform normal physiological activities [20,21]. The face of the upper facet of the CFJ is facing up and leaning backward. The articular surface of the axis is nearly horizontal, while the articular surface of the inferior articular process faces downward and leans forward to form an angle of approximately $40^{\circ}-45^{\circ}$ with the plane of the anterior vertebral body. In addition, the joint capsule of the CFJ is thin and relatively loose and can slide, and there is a lack of ligament fixation between the transverse processes of the cervical spine. Due to these special anatomical relationships, the cervical spine has good mobility and poor stability and is easily injured. In this study, after statistical analysis of all measured parameters, it was found that the cross-sectional space between the facet joints of each segment from C2-3 C6-7 gradually widened from top to bottom, the distance between C6-7 was the widest, the parameters of each segment were greater in males than females, and the difference between males and females was statistically significant. These results are similar to the results observed by Yoganandan et al. [1] in cadavers. On the sagittal plane, the upper and lower diameters of the CFJs from C2-3 C6-7 were significantly different between men and women. All parameters were greater in men than women. On the coronal plane, the left and right diameters of the facet joints were very similar. The left and right diameters of the CFJs from C2-3 C6-7 increase from top to bottom along the cervical spine. The left and right diameters of each segment were also significantly larger in men than women. This result is slightly different from that reported by Huang Yuanchi et al. [22]. We consider that this difference may be related to the measurement method and the method of selecting the measurement plane. We believe that an abnormal CFJ shape, anteroposterior diameter, gap distance, or left or right diameter may be important factors affecting the overall stability and mobility of the cervical spine.

Degenerative change is a natural phenomenon that occurs after the growth and development of the human body is stopped, and it cannot be avoided. The intervertebral disc is the first human tissue to undergo degenerative change. These degenerative changes may occur simultaneously or successively with degenerative changes in the CFJs and the surrounding ligaments.

A study by Stemper et al. [23] showed that degenerative changes in the cervical intervertebral disc led to a reduction in the height of the corresponding segment of the intervertebral space, which in turn caused a significant increase in the pressure on the facet joints, resulting in degenerative changes in the CFJs that can cause "cervical facet joint pain" over time. In addition, the CFJs constitute the posterior wall of the cervical intervertebral foramen, to which the front spinal nerve roots are adjacent. Degenerative changes in the facet joints can lead to stenosis of the cervical intervertebral foramen, causing compression of the spinal nerve roots and a series of clinical symptoms. This study found that there were often differences in the morphology and measured parameters of the left and right facet joints in the same segment in the same individual. Whether this difference is related to the degenerative changes in the CFJs needs further research.

At present, the most effective way to relieve the symptoms of nerve root compression caused by degenerative cervical spine disease is to enlarge the intervertebral foramen through surgery. The two most commonly used methods are to increase the gap between the facet joint interface of the posterior foramina of the diseased segment and restore the height between the upper and lower vertebral bodies of the corresponding segment [24]. The above two methods mostly involve traditional open surgery, with relatively large incisions, great tissue destruction, great intraoperative bleeding, and a long postoperative recovery time. A more ideal approach is to use a CFJ interface expander to horizontally and longitudinally expand the joint space of the cervical articular process through minimally invasive posterior cervical surgery. In this way, the intervertebral foramen space can be directly expanded, so that the compressed nerve roots can be fully and effectively decompressed, ultimately relieving the clinical symptoms. This operation is generally minimally invasive. The advantage of minimally invasive surgery compared with traditional open surgery is that minimally invasive surgery involves a relatively small incision, little damage to neck tissue, fast postoperative recovery, and little impact on postoperative cervical spine stability. Through the measurement and analysis of the 3D parameters of the CFJs, this study mainly hopes to provide detailed and reliable data for the production of a CFJ interface distractor. The design of the fusion cage should consider the shape, width, length, height, material, stability and bone graft window [25]. In the postproduction of CFJ interface distractors, based on the measured parameters provided in this study, CFJ interface distractors can be made with different specifications according to the sex of the patient and the segments involved to achieve better distraction and fusion effects, thereby guaranteeing the success of the minimally invasive surgical treatment of CFJs.

This study only measured CFJ data in a normal population; these data do not represent diseased populations. In addition, the sample size of the study was not very large, and all patients came from Xuzhou and surrounding areas. In the future, such work can be performed at multiple centres in multiple regions and larger areas. The measurements of samples can be compared with the anatomical measurements of physical specimens to increase the authenticity and reliability of the measured parameters. 
Funding Jiangsu Science and Technology Department Project (Social development-Clinical Frontier Technology, NO: BE2016647).

\section{Compliance with ethical standards}

Conflict of interest The authors declare that they have no conflict of interest.

Open Access This article is licensed under a Creative Commons Attribution 4.0 International License, which permits use, sharing, adaptation, distribution and reproduction in any medium or format, as long as you give appropriate credit to the original author(s) and the source, provide a link to the Creative Commons licence, and indicate if changes were made. The images or other third party material in this article are included in the article's Creative Commons licence, unless indicated otherwise in a credit line to the material. If material is not included in the article's Creative Commons licence and your intended use is not permitted by statutory regulation or exceeds the permitted use, you will need to obtain permission directly from the copyright holder. To view a copy of this licence, visit http://creativecommons.org/licenses/by/4.0/.

\section{References}

1. Yoganandan N, Knowles SA, Maiman DJ (2003) Anatomic study of the morphology of human cervical facet joint. Spine 28:2317-2323

2. Fu L, Ma J, Ma X, Sun L, Wang Y (2015) Progress in the biomechanics of facet joints. Chin J Orthop 9:970-974

3. Torg JS (1991) Athletic injuries to the head, neck, and face, 2nd edn. Mosby-Year Book, St. Louis, pp 372-373

4. Jia LS (2009) Cervical facet joint osteoarthritis. Chin J Spine Spinal Cord 19(1):72-73

5. Ngo LM, Aizawa T, Hoshikawa T et al (2012) Fracture and contralateral dislocation of the twin facet joints of the lower cervical spine. Eur Spine J 21(2):282-288

6. Gellhorn AC, Katz JN, Suri P (2013) Osteoarthritis of the spine: the facet joints. Nat Rev Rheumatol 9(4):216-224

7. Chung SB, Lee S, Kim H et al (2012) Significance of inter facet distance, facet joint orientation, and lumbar lordosis in spondylolysis. Clin Anat 25(3):391-397

8. Riley LH III, Vaccaro AR, Dettori JR, Hashimoto R (2010) Postoperative dysphagia in anterior cervical spine surgery. Spine 35(suppl):S76-S85

9. Mummaneni PV, Kaiser MG, Matz PG et al (2009) Cervical surgical techniques for the treatment of cervical spondylotic myelopathy. J Neurosurg Spine 11:130-141

10. Zeng H, Zou DW, Wu J (2012) Imaging observation and clinical significance of lower cervical facet joints. Chin J Spine Spinal Cord 22(1):59-64
11. Dalcanto RA, Lieberman I, Inceoglu S et al (2005) Biomechanical comparison of transarticular facet screws to lateral mass plates in two-level instrumentations of the cervical spine. Spine (Phila Pa 1976) 30(8):897-989

12. Xu R, Liu G, Zhao H (2010) The relationship between anatomical measurement of thoracic facet joints and transarticular screw fixation. Chin J Bone Joint Injury 25(6):481-483

13. Kettler A, Werner K, Wilke HJ (2007) Morphological changes of cervical facet joints in elderly individuals. Eur Spine J 7:987-992

14. Xi X, Wu Q, Tang H, Hu K, Li K, Li X, Chen L (2009) X-ray measurement data of the inclination of the lower cervical vertebrae and facet joints in adults. Chin Tissue Eng Res Clin Rehabil 13(4):662-666

15. Sun X, Cao L, Zhang L (1996) CT study of the morphology and horizontal inclination of the facet joints of the cervical spine. Chin J Radiol 5:51-53

16. Yang X, Chen F, Han P (2002) Simulation imaging. People's Medical Publishing House, Beijing

17. Li W, Li D, Duan L (2006) The diagnostic value of multi-slice spiral CT multiplanar reconstruction in the lumbar facet joint injuries. J Med Radiol Technol 252(8):57

18. Wang Q, Guo M, Xu S, Ma J, Meng L, Yang H, Liu J, Xiang L (2017) The clinical significance of measuring the facet joints of the lower cervical spine using image archiving and communication systems. J Spine Surg 15(3):171-176

19. Denis F (1983) The three column spine and its significance in the classification of acute thoracolumbar spinal injuries. Spine (Phila Pa 1976) 8(8):817-831

20. Dai LY (2001) Orientation and tropism of lumbar facet joints in degenerative spondylolisthesis. Int Orthop 25(1):40-42

21. Liu L, Wang X, Kang X, Li G, Li X, Gao S, Cai Y, Zhang S, Li Z (2020) Digital three-dimensional morphology study of the facet joints of the cervical spine in children aged 7-12 years. Chin Tissue Eng Res 24(6):877-881

22. Huang Y, Zou D, Wu J, Chen Z (2012) Application of threedimensional spiral CT measurement of cervical facet joints in digital orthopedics. Chin J Orthop 20(15):1405-1408

23. Stemper BD, Yoganandan N, Pinta FA (2005) Effects of abnormal posture on capsular ligament elongations in a computational model subjected to whiplash loading. Biomech J 38(6):1313-1323

24. Kettler A, Werner K, Wilke HJ (2007) Morphological changes of cervical facet joints in elderly individuals. Eur Spine $\mathbf{J}$ 16(7):987-992

25. Chong E, Pelletier MH, Mobbs RJ et al (2015) The design evolution of interbody cages in anterior cervical discectomy and fusion: a systematic review. BMC Musculoskelet Disord 16:99

Publisher's Note Springer Nature remains neutral with regard to jurisdictional claims in published maps and institutional affiliations. 